El ectron el ectron coal escence and count er bal ance functi ons for at ons

\begin{tabular}{|l|l|}
\hline 著者 & KOGA Toshi kat su \\
\hline $\begin{array}{l}\mathrm{j} \text { our nal or } \\
\text { publ i cat } \mathrm{i} \text { on } \mathrm{ti} t \mathrm{l} \text { e }\end{array}$ & The $\mathrm{j}$ ournal of chemical physics \\
\hline vol une & 114 \\
\hline number & 1 \\
\hline page $\mathrm{r}$ ange & $102-107$ \\
\hline year & $2001-01-01$ \\
\hline URL & ht $\mathrm{p}: / /$ hdl . handl e. net $/ 10258 / 791$ \\
\hline
\end{tabular}




\title{
Electron-electron coalescence and counterbalance functions for atoms
}

\author{
Toshikatsu Koga ${ }^{\text {a) }}$ \\ Department of Applied Chemistry, Muroran Institute of Technology, Muroran, Hokkaido 050-8585, Japan
}

(Received 28 September 2000; accepted 17 October 2000)

\begin{abstract}
For many-electron atoms, spherically averaged electron-electron coalescence $h_{0}(R)$ and counterbalance $d_{0}(u)$ functions are studied which, respectively, represent the probability densities that any electron pairs with zero interelectronic distance are located at a radius $R$ from the nucleus and that any electron pairs with zero center-of-mass radius have a relative distance $u$. For the exact and Hartree-Fock (HF) wave functions, cusp relations $h_{0}^{\prime}(0) / h_{0}(0)=-4 Z$ and $d_{0}^{\prime}(0) / d_{0}(0)$ $=-2 Z$ are derived theoretically, where the prime denotes the first derivative and $Z$ is nuclear charge. At the Hartree-Fock limit level, both functions $h_{0}(R)$ and $d_{0}(u)$ are found to be monotonically decreasing with a single maximum at $R=0$ or $u=0$ for all the 102 atoms He through Lr. The long-range asymptotic behavior of the coalescence and counterbalance functions is governed in general by the orbital energy of the highest occupied atomic orbital. (C) 2001 American Institute of Physics. [DOI: 10.1063/1.1331104]
\end{abstract}

\section{INTRODUCTION AND DEFINITIONS}

The motion of two particles in space is specified by the relative and center-of-mass coordinates. For an $N$-electron system $(N \geqslant 2)$, the probability density that any electron pairs have a relative vector $\mathbf{u}$ and a center-of-mass vector $\mathbf{R}$ is given by

$$
\begin{aligned}
\Gamma(\mathbf{u}, \mathbf{R}) & =\left\langle\sum_{i=1}^{N-1} \sum_{j=i+1}^{N} \delta\left[\mathbf{u}-\left(\mathbf{r}_{i}-\mathbf{r}_{j}\right)\right] \delta\left[\mathbf{R}-\left(\mathbf{r}_{i}+\mathbf{r}_{j}\right) / 2\right]\right\rangle \\
& =\left\langle\sum_{i=1}^{N-1} \sum_{j=i+1}^{N} \delta\left[\mathbf{r}_{i}-(\mathbf{R}+\mathbf{u} / 2)\right] \delta\left[\mathbf{r}_{j}-(\mathbf{R}-\mathbf{u} / 2)\right]\right\rangle,
\end{aligned}
$$

where $\delta(\mathbf{x})$ is the three-dimensional Dirac delta function and the angular brackets \langle\rangle stand for the expectation value over the wave function $\Psi\left(\mathbf{x}_{1}, \ldots, \mathbf{x}_{N}\right)$ with $\mathbf{x}_{i} \equiv\left(\mathbf{r}_{i}, \sigma_{i}\right)$ being the combined position-spin coordinates of the electron $i$. If we are interested in the relative motion of electron pairs, the integration of Eq. (1) over $\mathbf{R}$ yields the intracule (relative motion) density ${ }^{1-3}$

$$
I(\mathbf{u})=\int d \mathbf{R} \Gamma(\mathbf{u}, \mathbf{R}),
$$

whose spherical average is

$$
h(u)=(4 \pi)^{-1} \int d \Omega_{u} I(\mathbf{u}),
$$

where $\left(u, \Omega_{u}\right)$ is the polar coordinates of the vector $\mathbf{u}$. The intracule densities $I(\mathbf{u})$ and $h(u)$ have been used in several physical and chemical contexts particularly in relation to the electron correlation problem (see Refs. 2-7 and the references therein). If we are concerned with the center-of-mass

${ }^{a)}$ Electronic mail: koga@mmm.muroran-it.ac.jp motion of electron pairs, on the other hand, the integration of Eq. (1) over u gives the extracule (center-of-mass motion) density $^{1-3}$

$$
E(\mathbf{R})=\int d \mathbf{u} \Gamma(\mathbf{u}, \mathbf{R}),
$$

whose spherical average is

$$
d(R)=(4 \pi)^{-1} \int d \Omega_{R} E(\mathbf{R}),
$$

where $\left(R, \Omega_{R}\right)$ is the polar coordinates of the vector $\mathbf{R}$. The extracule densities $E(\mathbf{R})$ and $d(R)$ were used to study the shell structure in some atoms and bonding characteristics in simple molecules (see Refs. 2, 3, 8-11 and the references therein).

A special value $I(\mathbf{0})=h(0)$ of the intracule densities is known $^{12-16}$ as the electron-electron coalescence density, which is the probability density of finding any two electrons $i$ and $j$ precisely at the same position in three-dimensional space or $\mathbf{r}_{i}=\mathbf{r}_{j}$. The coalescence density appears in the evaluation of the relativistic ${ }^{17}$ and radiative ${ }^{18}$ corrections for atoms and molecules. The coalescence or Fermi hole (i.e., zero probability density) exists for two electrons with the same spin. Correspondingly, a special value $E(\mathbf{0})=d(0)$ of the extracule densities is known ${ }^{15,16,19}$ as the electronelectron counterbalance density, which represents the probability density of finding any two electrons $i$ and $j$ exactly at the opposite positions with respect to the coordinate origin (i.e., the nucleus in atoms) or $\mathbf{r}_{i}=-\mathbf{r}_{j}$ in three-dimensional space. In the Hartree-Fock theory, the presence of electronelectron counterbalance holes is known ${ }^{20}$ between two electrons in spin-orbitals with the same spin and the same spatial inversion symmetry.

In the present paper, we study the mathematical structure and properties of electron-electron coalescence $I_{0}(\mathbf{R})$ and 
counterbalance $E_{0}(\mathbf{u})$ functions, as well as their spherical averages $h_{0}(R)$ and $d_{0}(u)$, which appear as two particular cases of the electron-pair density $\Gamma(\mathbf{u}, \mathbf{R})$ given by Eq. (1)

$$
\begin{aligned}
& I_{0}(\mathbf{R})=\Gamma(\mathbf{0}, \mathbf{R}), \\
& h_{0}(R)=(4 \pi)^{-1} \int d \Omega_{R} I_{0}(\mathbf{R})
\end{aligned}
$$

and

$$
\begin{aligned}
& E_{0}(\mathbf{u})=\Gamma(\mathbf{u}, \mathbf{0}), \\
& d_{0}(u)=(4 \pi)^{-1} \int d \Omega_{u} E_{0}(\mathbf{u}) .
\end{aligned}
$$

The coalescence functions $I_{0}(\mathbf{R})$ and $h_{0}(R)$ describe the spatial distribution of electron pairs with zero relative vector (or distance) as a function of the center-of-mass vector $\mathbf{R}$ or its radius $R$. By definitions, we have

$$
\int d \mathbf{R} I_{0}(\mathbf{R})=4 \pi \int_{0}^{\infty} d R R^{2} h_{0}(R)=I(\mathbf{0})=h(0),
$$

which implies that the coalescence functions detail out the spatial origin of the electron-electron coalescence density $I(\mathbf{0})=h(0)$. The coalescence function $I_{0}(\mathbf{R})$ has been used $^{21-24}$ in the analysis of some density functional approximations. Analogously, the counterbalance functions $E_{0}(\mathbf{u})$ and $d_{0}(u)$ represent the distribution of electron pairs with zero center-of-mass vector (or radius) as a function of the relative vector $\mathbf{u}$ or its magnitude $u$. We immediately find

$$
\int d \mathbf{u} E_{0}(\mathbf{u})=4 \pi \int_{0}^{\infty} d u u^{2} d_{0}(u)=E(\mathbf{0})=d(0),
$$

and that the counterbalance functions clarify the spatial origin of the electron-electron counterbalance density $E(\mathbf{0})$ $=d(0)$. In the next section, we examine the mathematical structure of the coalescence and counterbalance functions for atoms mainly in the Hartree-Fock theory. It will be found that these functions satisfy cusp conditions at $R=0$ or $u$ $=0$ characterized by nuclear charge $Z$. The long-range asymptotic behavior of the functions, on the other hand, is governed by the orbital energy of the highest occupied orbital. In Sec. III, the numerical results are presented and discussed for the 102 atoms from $\mathrm{He}(Z=2)$ to $\operatorname{Lr}(Z$ $=103$ ) based on the numerical Hartree-Fock calculations. Hartree atomic units are used throughout.

\section{MATHEMATICAL STRUCTURE OF COALESCENCE AND COUNTERBALANCE FUNCTIONS}

\section{A. Electron-electron coalescence function}

The electron-electron coalescence function $I_{0}(\mathbf{R})$, defined by Eq. (4a), is explicitly written as

$$
I_{0}(\mathbf{R})=\left\langle\sum_{i=1}^{N-1} \sum_{j=i+1}^{N} \delta\left(\mathbf{r}_{i}-\mathbf{R}\right) \delta\left(\mathbf{r}_{j}-\mathbf{R}\right)\right\rangle,
$$

$$
\begin{aligned}
I_{0}(\mathbf{R})= & \left(\begin{array}{c}
N \\
2
\end{array}\right) \int d \sigma_{1} d \sigma_{2} d \mathbf{x}_{3} \cdots d \mathbf{x}_{N} \\
& \times\left|\Psi\left(\mathbf{R}, \sigma_{1}, \mathbf{R}, \sigma_{2}, \mathbf{x}_{3}, \ldots, \mathbf{x}_{N}\right)\right|^{2} .
\end{aligned}
$$

For a small value of $r_{i}=\left|\mathbf{r}_{i}\right|$, Bingel showed ${ }^{25}$ that Kato's cusp condition, ${ }^{26}$ due to the electron-nucleus Coulomb singularity in the Schrödinger equation, implies

$$
\begin{aligned}
& \begin{aligned}
\Psi\left(\mathbf{r}_{1}, \ldots, \mathbf{r}_{N}\right)= & \Psi\left(\mathbf{r}_{1}, \ldots, \mathbf{r}_{i-1}, \mathbf{0}, \mathbf{r}_{i+1}, \ldots, \mathbf{r}_{N}\right)\left(1-Z r_{i}\right) \\
& +\mathbf{r}_{i} \cdot \mathbf{a}_{i}+O\left(r_{i}^{2}\right)
\end{aligned} \\
& \mathbf{a}_{i}=\mathbf{a}_{i}\left(\mathbf{r}_{1}, \ldots, \mathbf{r}_{i-1}, \mathbf{r}_{i+1}, \ldots, \mathbf{r}_{N}\right)
\end{aligned}
$$

where the spin variables have been suppressed. Combining Eq. (9) for $i=1$ and $i=2$ and putting $\mathbf{r}_{1}= \pm \mathbf{r}_{2}=\mathbf{r}$, we have

$$
\begin{aligned}
\Psi\left(\mathbf{r}, \pm \mathbf{r}, \mathbf{r}_{3}, \ldots, \mathbf{r}_{N}\right)= & \Psi\left(\mathbf{0}, \mathbf{0}, \mathbf{r}_{3}, \ldots, \mathbf{r}_{N}\right)(1-2 Z r) \\
& +\mathbf{r} \cdot\left[\mathbf{a}_{1}\left(\mathbf{0}, \mathbf{r}_{3}, \ldots, \mathbf{r}_{N}\right)\right. \\
& \left. \pm \mathbf{a}_{2}\left(\mathbf{0}, \mathbf{r}_{3}, \ldots, \mathbf{r}_{N}\right)\right]+O\left(r^{2}\right) .
\end{aligned}
$$

Substituting Eq. (9c) with the plus sign into Eq. (8b) and taking the spherical average of Eq. (8b), we obtain

$$
h_{0}(R)=C(1-4 Z R)+O\left(R^{2}\right),
$$

where

$$
\begin{aligned}
C= & (4 \pi)^{-1}\left(\begin{array}{c}
N \\
2
\end{array}\right) \int d \sigma_{1} d \sigma_{2} d \mathbf{x}_{3} \cdots d \mathbf{x}_{N} \\
& \times\left|\Psi\left(\mathbf{0}, \sigma_{1}, \mathbf{0}, \sigma_{2}, \mathbf{x}_{3}, \ldots, \mathbf{x}_{N}\right)\right|^{2} .
\end{aligned}
$$

Thus the exact electron-electron coalescence function $h_{0}(R)$ satisfies a cusp relation

$$
h_{0}^{\prime}(0) / h_{0}(0)=-4 Z \text {, }
$$

where the prime means the first derivative. The right-handside of Eq. (10c) is precisely twice the value of the electronnucleus cusp constant $(-2 Z)$ known $^{25-27}$ for the singleelectron density $\rho(r)$.

For a single determinant wave function composed of a set of orthonormal spin-orbitals $\psi_{i}(\mathbf{r}) \eta_{i}(\sigma)$, the CondonSlater rules (see, e.g., Ref. 28) rearrange Eq. (8a) as

$$
I_{0}(\mathbf{R})=\sum_{i=1}^{N-1} \sum_{j=i+1}^{N}\left[1-\delta_{s}(i, j)\right]\left|\psi_{i}(\mathbf{R})\right|^{2}\left|\psi_{j}(\mathbf{R})\right|^{2},
$$

where $\delta_{s}(i, j)$ is unity if the spin-orbitals $i$ and $j$ have the same spin and is zero if they have the opposite spins. Namely, the coalescence function $I_{0}(\mathbf{R})$ is the sum of products of two orbital densities $\left|\psi_{i}(\mathbf{r})\right|^{2}$ and $\left|\psi_{j}(\mathbf{r})\right|^{2}$. Moreover, two electrons in spin-orbitals with parallel spins do not contribute to the coalescence function.

For atomic systems, we can generally assume that the single-electron spatial function $\psi_{i}(\mathbf{r})$ is expressed by a product of the radial $R_{n l}(r)$ and spherical harmonic $Y_{l m}\left(\Omega_{r}\right)$ functions, where $n, l$, and $m$ denote the principal, azimuthal, and magnetic quantum numbers, respectively. Then the spherical average $h_{0}(R)$ of the coalescence function is obtained as 


$$
h_{0}(R)=\sum_{i=1}^{N-1} \sum_{j=i+1}^{N}\left[1-\delta_{s}(i, j)\right] A_{i j}\left|R_{i}(R)\right|^{2}\left|R_{j}(R)\right|^{2}
$$

where

$$
\begin{aligned}
A_{i j} & =(4 \pi)^{-1} \int d \Omega\left|Y_{l_{i} m_{i}}(\Omega)\right|^{2}\left|Y_{l_{j} m_{j}}(\Omega)\right|^{2} \\
& =(4 \pi)^{-2} \sum_{l=0}^{\min \left(2 l_{i}+2 l_{j}\right)}(2 l+1) a^{l}\left(l_{i} m_{i} ; l_{j} m_{j}\right),
\end{aligned}
$$

in which $a^{k}\left(l m ; l^{\prime} m^{\prime}\right)$ is the Condon-Shortley parameter. ${ }^{29}$ Some explicit values of $4 \pi A_{i j}$ can be found in Ref. 16.

For a small value of $r$, the radial function $R_{n l}(r)$ is expanded $^{30}$ as

$$
R_{n l}(r)=c_{n l} r^{l}\left[1-\frac{Z}{l+1} r+O\left(r^{2}\right)\right]
$$

where $c_{n l}$ is a nonzero real constant. We then obtain

$$
\begin{aligned}
\left|R_{i}(r)\right|^{2}\left|R_{j}(r)\right|^{2}= & c_{n_{i} l_{i}}^{2} c_{n_{j} l_{j}}^{2} r^{2 l_{i}+2 l_{j}} \\
& \times\left[1-2 Z\left(\frac{1}{l_{i}+1}+\frac{1}{l_{j}+1}\right) r+O\left(r^{2}\right)\right] .
\end{aligned}
$$

Therefore, we find that the leading contribution to the coalescence function $h_{0}(R)$ for a small $R$ comes from combinations of $s$-type orbitals and

$$
h_{0}(R)=C_{\mathrm{HF}}(1-4 Z R)+O\left(R^{2}\right),
$$

where

$$
C_{\mathrm{HF}}=\sum_{i=1}^{N-1} \sum_{j=i+1}^{N} \delta_{l_{i} 0} \delta_{l_{j} 0}\left[1-\delta_{s}(i, j)\right] A_{i j} c_{n_{i} 0}^{2} c_{n_{j} 0}^{2},
$$

in which $\delta_{i j}$ is the Kronecker's delta. From Eq. (14a), we immediately find that the Hartree-Fock coalescence function $h_{0}(R)$ also satisfies the cusp relation given by Eq. (10c).

For a large value of $r$, the radial function $R_{n l}(r)$ has $^{31-33}$ an asymptotic decay

$$
R_{n l}(r) \approx \exp \left(-\sqrt{-2 \varepsilon_{h}} r\right),
$$

in general, where $\varepsilon_{h}$ is the orbital energy of the highest occupied atomic orbital. An exception is

$$
R_{n l}(r) \approx \exp \left(-\sqrt{-2 \varepsilon_{n l}} r\right),
$$

when only $s$ orbitals are occupied. As long as the groundstate neutral atoms are concerned, we thus obtain the longrange asymptotic behavior of $h_{0}(R)$ as

$h_{0}(R)$

$$
\approx\left\{\begin{array}{l}
\exp \left[-2\left(\sqrt{-2 \varepsilon_{1 s}}+\sqrt{-2 \varepsilon_{2 s}}\right) R\right], \text { for the } \mathrm{Li} \text { atom } \\
\exp \left(-4 \sqrt{-2 \varepsilon_{h}} R\right), \quad \text { otherwise }
\end{array} .\right.
$$

\section{B. Electron-electron counterbalance function}

From Eqs. (1) and (5a), the electron-electron counterbalance function $E_{0}(\mathbf{u})$ reads

$$
E_{0}(\mathbf{u})=\left\langle\sum_{i=1}^{N-1} \sum_{j=i+1}^{N} \delta\left(\mathbf{r}_{i}-\mathbf{u} / 2\right) \delta\left(\mathbf{r}_{j}+\mathbf{u} / 2\right)\right\rangle,
$$

or

$$
\begin{aligned}
E_{0}(\mathbf{u})= & \left(\begin{array}{c}
N \\
2
\end{array}\right) \int d \sigma_{1} d \sigma_{2} d \mathbf{x}_{3} \cdots d \mathbf{x}_{N} \\
& \times\left|\Psi\left(\frac{\mathbf{u}}{2}, \sigma_{1},-\frac{\mathbf{u}}{2}, \sigma_{2}, \mathbf{x}_{3}, \ldots, \mathbf{x}_{N}\right)\right|^{2} .
\end{aligned}
$$

If we apply Eq. (9c) with the minus sign to Eq. (16b) and take the spherical average of Eq. (16b), we then obtain

$$
d_{0}(u)=C(1-2 Z u)+O\left(u^{2}\right),
$$

where the constant $C$ is defined by Eq. (10b). From Eq. (17a), a cusp relation

$$
d_{0}^{\prime}(0) / d_{0}(0)=-2 Z
$$

follows immediately for the exact electron-electron counterbalance function $d_{0}(u)$. Further, comparison of Eqs. (10a) and (17a) gives

$$
h_{0}(0)=d_{0}(0)=C,
$$

corresponding to the probability density that any two electrons are coalescent at the nuclear position.

For single determinant wave functions, Eq. (16a) is rewritten as

$$
\begin{aligned}
E_{0}(\mathbf{u})= & \sum_{i=1}^{N-1} \sum_{j=i+1}^{N} \psi_{i}^{*}(\mathbf{u} / 2) \psi_{j}^{*}(-\mathbf{u} / 2) \\
& \times\left[\psi_{i}(\mathbf{u} / 2) \psi_{j}(-\mathbf{u} / 2)-\delta_{s}(i, j) \psi_{i}(-\mathbf{u} / 2) \psi_{j}(\mathbf{u} / 2)\right] .
\end{aligned}
$$

If the orbital $\psi_{i}(\mathbf{r})$ has spatial inversion symmetry $\psi_{i}(-\mathbf{r})$ $=(-1)^{\lambda_{i}} \psi_{i}(\mathbf{r})$ specified by an index $\lambda_{i}$, Eq. (19a) is simplified to

$$
\begin{aligned}
E_{0}(\mathbf{u})= & \sum_{i=1}^{N-1} \sum_{j=i+1}^{N}\left[1-\delta_{s}(i, j)(-1)^{\lambda_{i}+\lambda_{j}}\right] \\
& \times\left|\psi_{i}(\mathbf{u} / 2)\right|^{2}\left|\psi_{j}(\mathbf{u} / 2)\right|^{2} .
\end{aligned}
$$

Apart from the factor $(-1)^{\lambda_{i}+\lambda_{j}}$, Eq. (19b) for the counterbalance function is analogous to Eq. (11) for the coalescence function, and $E_{0}(\mathbf{u})$ is also the sum of products of orbital densities $\left|\psi_{i}(\mathbf{u} / 2)\right|^{2}$ and $\left|\psi_{j}(\mathbf{u} / 2)\right|^{2}$. We note that two electrons in spin-orbitals with the same spin and the same inversion symmetry give no contribution to the counterbalance function.

For atomic system with $\psi_{i}(\mathbf{r})=R_{n_{i} l_{i}}(r) Y_{l_{i} m_{i}}\left(\Omega_{r}\right)$, the azimuthal quantum number $l_{i}$ plays a role of the inversion symmetry index $\lambda_{i}$, and the spherically averaged counterbalance function $d_{0}(u)$ is obtained to be

$$
\begin{aligned}
d_{0}(u)= & \sum_{i=1}^{N-1} \sum_{j=i+1}^{N}\left[1-\delta_{s}(i, j)(-1)^{l_{i}+l_{j}}\right] \\
& \times A_{i j}\left|R_{i}(u / 2)\right|^{2}\left|R_{j}(u / 2)\right|^{2},
\end{aligned}
$$

where $A_{i j}$ is defined by Eq. (12b). 
Exactly the same discussion as the coalescence function $h_{0}(R)$ applies to the small- $u$ behavior of the Hartree-Fock $d_{0}(u)$, leading to the short-range expansion of the electronelectron counterbalance function:

$$
d_{0}(u)=C_{\mathrm{HF}}(1-2 Z u)+O\left(u^{2}\right),
$$

where the constant $C_{\mathrm{HF}}$ is given by Eq. (14b). We, therefore, find that the cusp condition given by Eq. (17b) also holds for the Hartree-Fock counterbalance function. Equation (18) is also valid provided that the constant $C$ is replaced with $C_{\mathrm{HF}}$. For the long-range asymptotic behavior of the counterbalance function $d_{0}(u)$, we obtain

$d_{0}(u) \approx\left\{\begin{array}{l}\exp \left[-\left(\sqrt{-2 \varepsilon_{1 s}}+\sqrt{-2 \varepsilon_{2 s}}\right) u\right], \text { for the Li atom } \\ \exp \left(-2 \sqrt{-2 \varepsilon_{h}} u\right), \quad \text { otherwise }\end{array}\right.$.

\section{Approximate isomorphism}

The relative and center-of-mass motions of two particles are completely independent. For an atomic system, however, comparison of the Hartree-Fock expressions for $h_{0}(R)$ and $d_{0}(u)$, Eqs. (12a) and (20), suggests an approximate isomorphism

$$
h_{0}(R) \cong d_{0}(2 R),
$$

between the coalescence and counterbalance functions, provided that the contributions of spin-orbital pairs with the same spin and $l_{i}+l_{j}=$ odd are small. The equality in Eq. (23) is rigorous for $R=0$ as shown by Eq. (18). All the cusp relations [Eqs. (10c) and (17b)], short-range expansions [Eqs. (10a), (14a), (17a), and (21a)], and long-range behaviors [Eqs. (15c) and (22)] of $h_{0}(R)$ and $d_{0}(u)$ are consistent with the relation (23). When Eq. (23) is combined with Eqs. (6) and (7), we have

$$
d(0) \cong 8 h(0),
$$

which was reported previously. ${ }^{15,16}$ If we introduce coalescence $\left\langle R^{n}\right\rangle$ and counterbalance $\left\langle u^{n}\right\rangle$ moments defined by

$$
\begin{aligned}
& \left\langle R^{n}\right\rangle=4 \pi \int_{0}^{\infty} d R R^{n+2} h_{0}(R) / h(0), \\
& \left\langle u^{n}\right\rangle=4 \pi \int_{0}^{\infty} d u u^{n+2} d_{0}(u) / d(0),
\end{aligned}
$$

then Eqs. (23) and (24) suggest an approximate proportionality relation

$$
\left\langle u^{n}\right\rangle \cong 2^{n}\left\langle R^{n}\right\rangle
$$

between the two sets of moments.

\section{NUMERICAL RESULTS FOR ATOMS HE THROUGH LR}

Using Eqs. (12a) and (20), we have calculated the accurate Hartree-Fock values of the electron-electron coalescence $h_{0}(R)$ and counterbalance $d_{0}(u)$ functions, as well as the associated moments $\left\langle R^{n}\right\rangle$ and $\left\langle u^{n}\right\rangle$, for the 102 neutral atoms from $\mathrm{He}(Z=2)$ to $\operatorname{Lr}(Z=103)$. For all the atoms, the experimental ground electronic configurations and $L S$ terms $^{34,35}$ were considered. The Hartree-Fock radial func-

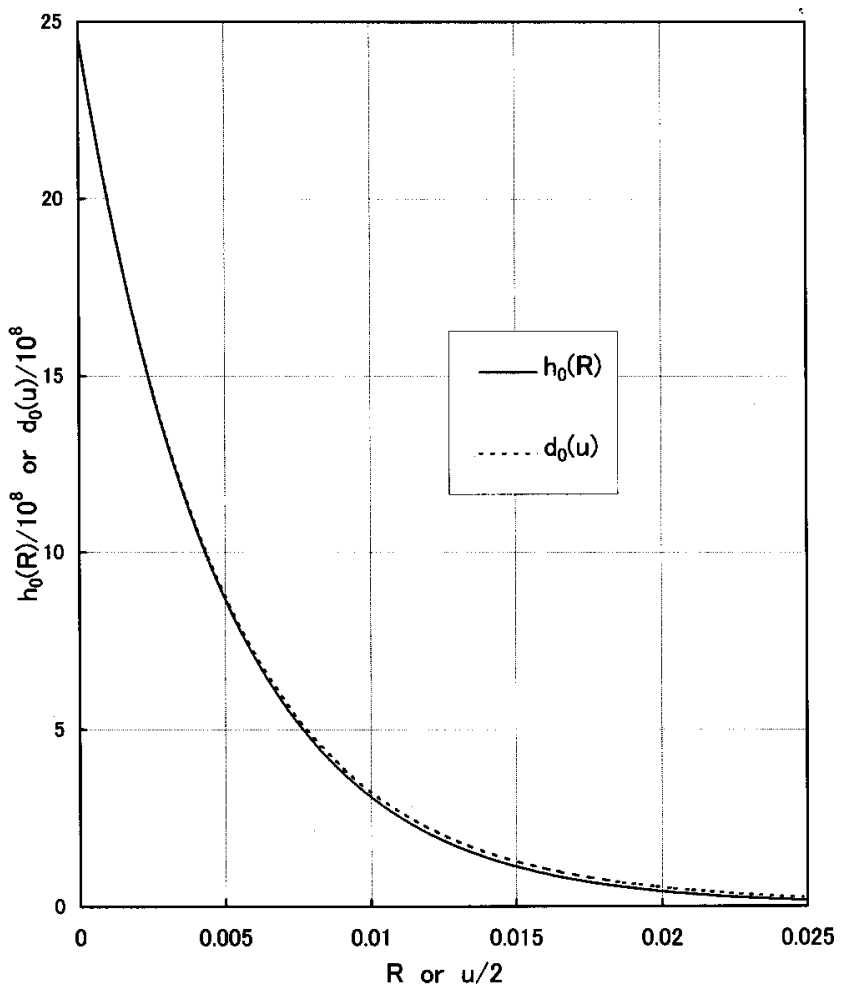

FIG. 1. The electron-electron coalescence $h_{0}(R)$ and counterbalance $d_{0}(u)$ functions for the Te atom $(Z=52)$.

tions $R_{n l}(r)$ were generated by the numerical Hartree-Fock method ${ }^{36,37}$ based on the MCHF72 program. ${ }^{38}$ The discretization of the radial variable $r$ was performed by $r_{i}=\exp \left[x_{\min }\right.$ $+(i-1) h] / Z\left(i=1,2, \ldots, N_{p}\right)$, where $x_{\min }=-6, h=3 / 100$, and $N_{p}=2^{9}=512$. The moments were computed by numerical integrations.

Examination of the electron-electron coalescence functions $h_{0}(R)$ for the 102 atoms shows that all the functions are monotonically decreasing with increasing $R$; the coalescent electron pairs are most likely at the nuclear position. The result is mainly due to the predominant contribution of the innermost $1 s$ orbital, as expected from Eq. (12a). Moreover, the coalescence function $h_{0}(R)$ is more condensed around the nucleus as the nuclear charge $Z$ increases. The cusp relation, Eq. (10c), is precisely fulfilled, since the numerical Hartree-Fock procedure ${ }^{37,38}$ imposes Eq. (13a) in the construction of atomic radial functions $R_{n l}(r)$. Exactly the same is true for the counterbalance functions $d_{0}(u)$ of the 102 atoms; $d_{0}(u)$ is a unimodal function with a maximum at $u=0$ and satisfies the cusp relation (17b). An example of the functions $h_{0}(R)$ and $d_{0}(u)$ is given in Fig. 1 for the Te atom $(Z=52)$ which lies approximately at the center of the 102 atoms examined. Figure 1 also exemplifies the approximate isomorphism $h_{0}(R) \cong d_{0}(2 R)$ between the coalescence and counterbalance functions observed in all the atoms. In particular, the equality is rigorous for the first three atoms $\mathrm{He}, \mathrm{Li}$, and $\mathrm{Be}$, where only $s$ orbitals are occupied.

The peak value $h_{0}(0)=d_{0}(0)$ of the coalescence and counterbalance functions corresponds to the probability density for the electron-electron-nucleus coalescence. Figure 2 


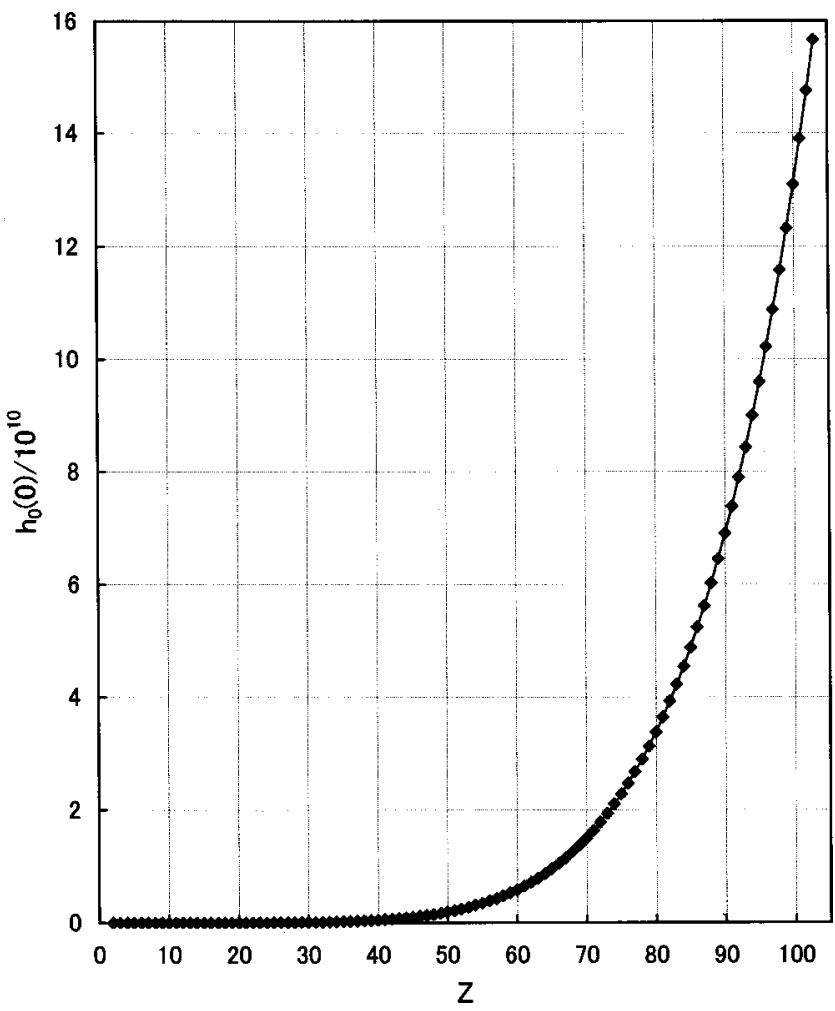

FIG. 2. The Z-dependence of the electron-electron-nucleus coalescence density $h_{0}(0)=d_{0}(0)$.

plots $h_{0}(0)$ as a function of $Z$. If we consider two electrons in an unscreened hydrogenic $1 s$ orbital with nuclear charge $Z$, the $h_{0}(0)$ value is found from Eq. (12a) to be proportional to $Z^{6}$. On the other hand, our regression analysis of the data in Fig. 2 gives $h_{0}(0) \cong 0.06422 Z^{6.164}$ with a correlation coefficient 1.0000. The result again supports the significance of two electrons in the $1 s$ orbital for the electron-electron coalescence and counterbalance functions.

The first moment $\langle R\rangle$ of the coalescence function $h_{0}(R)$ represents the average radius of coalescent electron pairs from the nucleus. As shown in Fig. 3, the average radius $\langle R\rangle$ decreases monotonically with increasing $Z$ or nuclear attraction; it is maximum ( 0.424 bohrs) at $Z=2$ and minimum $(0.024$ bohrs) at $Z=103$. The hydrogenic $1 s$ model predicts that $\langle R\rangle$ is proportional to $Z^{-1}$. However, a regression analysis yields $\langle R\rangle \cong 0.4747 Z^{-0.6514}$ with a correlation coefficient 0.9965 , and the contributions of coalescent electron pairs other than the $1 s$ electrons are not negligible. The corresponding moment $\langle u\rangle$ of the counterbalance function $d_{0}(u)$ represents the average interelectronic distance of counterbalanced electrons. Figure 3 shows that as $Z$ increases, the average distance $\langle u\rangle$ decreases monotonically with the maximum 0.849 bohrs at $Z=2$ and the minimum 0.061 bohrs at $Z=103$. The increased nuclear binding reduces the relative distance of the counterbalanced electrons. As in the case of $\langle R\rangle$, the hydrogenic $1 s$ model predicts $Z^{-1}$ dependence for $\langle u\rangle$, but our regression analysis results in $\langle u\rangle \cong 1.003 Z^{-0.6088}$ with a correlation coefficient 0.9980 . We have also examined the ratio $\langle u\rangle /\langle R\rangle$ between the two average distances. The ratio is 2 precisely for the first three at-

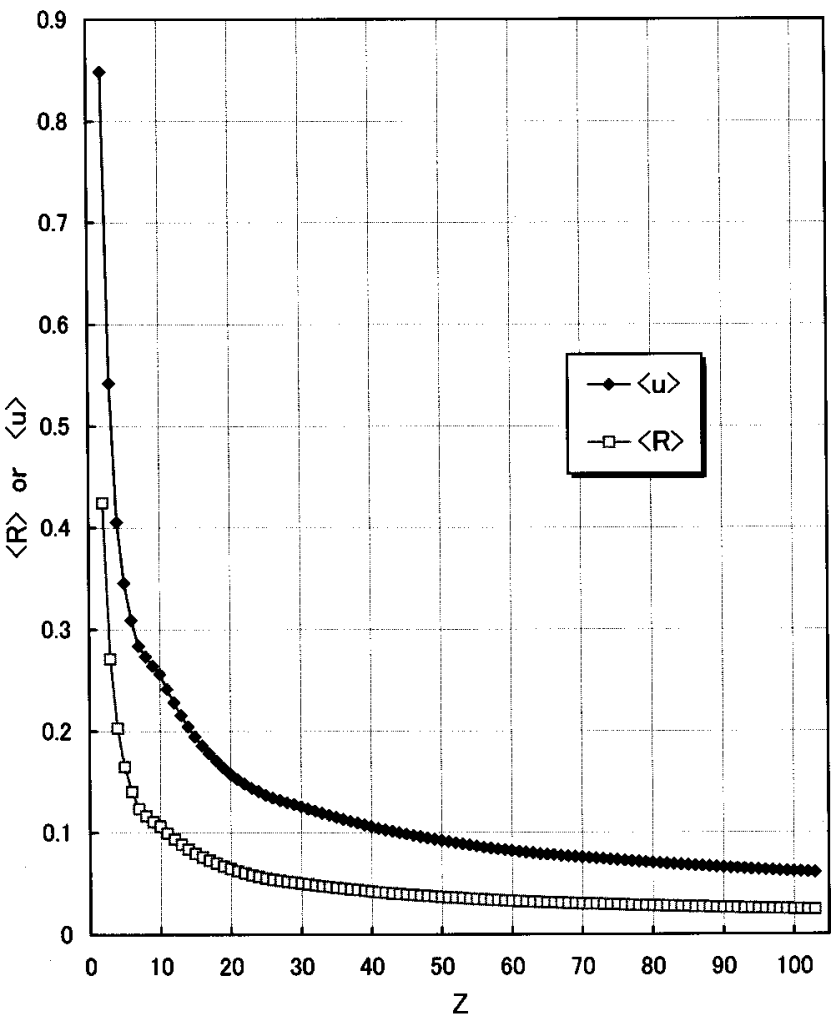

FIG. 3. The $Z$-dependence of the average radius $\langle R\rangle$ of the coalescent electrons and of the average interelectronic distance $\langle u\rangle$ of the counterbalanced electrons.

oms, but is always greater than 2 for the remaining 99 atoms. The average over the 102 atoms is 2.48 , and the approximate proportionality relation, Eq. (26), is not very accurate.

\section{SUMMARY}

Mathematical structure of the spherically averaged electron-electron coalescence $h_{0}(R)$ and counterbalance $d_{0}(u)$ functions has been studied for many-electron atoms. In both the exact and Hartree-Fock frameworks, the shortrange behaviors of the functions $h_{0}(R)$ and $d_{0}(u)$ have been clarified. In particular, the cusp relations $h_{0}^{\prime}(0) / h_{0}(0)$ $=-4 Z$ and $d_{0}^{\prime}(0) / d_{0}(0)=-2 Z$ have been derived theoretically. An approximate isomorphic relation $h_{0}(R) \cong d_{0}(2 R)$ has also been obtained. Numerical examination of the 102 neutral atoms from $\mathrm{He}$ to $\mathrm{Lr}$ has shown that both the coalescence $h_{0}(R)$ and counterbalance $d_{0}(u)$ functions are monotonically decreasing with a single maximum at $R=0$ or $u$ $=0$ for all the cases, due to the predominant contribution of the innermost $1 s$ electrons. The $Z$-dependence of the electron-electron-nucleus coalescence density $h_{0}(0)$ $=d_{0}(0)$, the average radius $\langle R\rangle$ of the coalescent electrons, and the average interelectronic distance $\langle u\rangle$ of the counterbalanced electrons has also been discussed.

\footnotetext{
${ }^{1}$ A. J. Coleman, Int. J. Quantum Chem., Symp. 1, 457 (1967).

${ }^{2}$ A. J. Thakkar, in Density Matrices and Density Functionals, edited by R. M. Erdahl and V. H. Smith, Jr. (Reidel, Dordrecht, 1987), pp. 553-581.

${ }^{3}$ R. J. Boyd and J. M. Ugalde, in Computational Chemistry, Part A, edited by S. Fraga (Elsevier, Amsterdam, 1992), pp. 273-299.

${ }^{4}$ P. E. Regier and A. J. Thakkar, J. Phys. B 17, 3391 (1984).
} 
${ }^{5}$ T. Koga and H. Matsuyama, J. Chem. Phys. 107, 8510 (1997).

${ }^{6}$ H. Matsuyama, T. Koga, E. Romera, and J. S. Dehesa, Phys. Rev. A 57, 1759 (1998).

${ }^{7}$ A. Sarsa, F. J. Galvez, and E. Buendia, J. Chem. Phys. 109, 7075 (1998).

${ }^{8}$ A. J. Thakkar and N. J. Moore, Int. J. Quantum Chem., Symp. 15, 393 (1981).

${ }^{9}$ T. Koga and H. Matsuyama, J. Chem. Phys. 108, 3424 (1998).

${ }^{10}$ T. Koga, H. Matsuyama, E. Romera, and J. S. Dehesa, Phys. Rev. A 57, 4212 (1998).

${ }^{11}$ F. J. Galvez, E. Buendia, and A. Sarsa, J. Chem. Phys. 111, 3319 (1999).

${ }^{12}$ J. S. Dehesa, J. C. Angulo, T. Koga, and K. Matsui, Phys. Rev. A 47, 5202 (1993).

${ }^{13}$ T. Koga, J. C. Angulo, and J. S. Dehesa, Proc. Indian Acad. Sci. (Chem. Sci.) 106, 123 (1994).

${ }^{14}$ J. M. Ugalde and C. Sarasola, Phys. Rev. A 49, 3081 (1994).

${ }^{15}$ T. Koga and H. Matsuyama, J. Chem. Phys. 107, 10062 (1997).

${ }^{16}$ T. Koga and H. Matsuyama, J. Phys. B 30, 5631 (1997).

${ }^{17}$ H. A. Bethe and E. Salpeter, Quantum Mechanics of One- and TwoElectron Atoms (Plenum, New York, 1977), p. 180.

${ }^{18}$ P. K. Kabir and E. Salpeter, Phys. Rev. 108, 1256 (1957).

${ }^{19}$ J. M. Mercero, J. E. Fowler, C. Sarasola, and J. M. Ugalde, Phys. Rev. A 59, 4255 (1999).

${ }^{20}$ T. Koga, J. Chem. Phys. 108, 2515 (1998).

${ }^{21}$ J. P. Perdew, A. Savin, and K. Burke, Phys. Rev. A 51, 4531 (1995).

${ }^{22}$ J. P. Perdew, M. Ernzerhof, K. Burke, and A. Savin, Int. J. Quantum Chem. 61, 197 (1997).

${ }^{23}$ K. Burke, J. P. Perdew, and M. Emzerhof, J. Chem. Phys. 109, 3760 (1998).
${ }^{24}$ J. Gräfenstein, A. M. Hjerpe, E. Kraka, and D. Cremer, J. Phys. Chem. A 104, 1748 (2000).

${ }^{25}$ W. A. Bingel, Z. Naturforsch. A 18, 1249 (1963).

${ }^{26}$ T. Kato, Commun. Pure Appl. Math. 10, 151 (1957).

${ }^{27}$ E. Steiner, J. Chem. Phys. 39, 2365 (1963).

${ }^{28}$ I. N. Levine, Quantum Chemistry, 5th ed. (Prentice Hall, Upper Saddle River, NJ, 2000), pp. 339-342.

${ }^{29}$ E. U. Condon and G. H. Shortley, The Theory of Atomic Spectra (Cambridge University Press, London, 1970), pp. 175-176.

${ }^{30}$ D. P. Chong, J. Chem. Phys. 47, 4907 (1967).

${ }^{31}$ N. C. Handy, M. T. Marron, and H. J. Silverstone, Phys. Rev. 180, 45 (1969).

${ }^{32}$ G. S. Handler, D. W. Smith, and H. J. Silverstone, J. Chem. Phys. 73, 3936 (1980).

${ }^{33}$ T. Ishida and K. Ohno, Theor. Chim. Acta 81, 355 (1992).

${ }^{34}$ C. E. Moore, Ionization Potentials and Ionization Limits Derived from the Analysis of Optical Spectra, NSRDS-NBS 34 (Nat. Bur. Stand. U.S., Washington, D.C., 1970).

${ }^{35}$ H. L. Anderson, A Physicist's Desk Reference (AIP Press, New York, 1989), p. 94.

${ }^{36}$ D. R. Hartree, The Calculation of Atomic Structure (Wiley, London, 1957).

${ }^{37}$ C. Froese-Fischer, The Hartree-Fock Method for Atoms (Wiley, New York, 1977).

${ }^{38}$ C. Froese-Fischer, Comput. Phys. Commun. 4, 107 (1972). 